International Journal of Pure and Applied Mathematics

Volume 100 No. 1 2015, 59-74

ISSN: 1311-8080 (printed version); ISSN: 1314-3395 (on-line version)

url: http://www.ijpam.eu

doi: http://dx.doi.org/10.12732/ijpam.v100i1.6

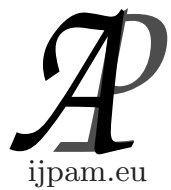

\title{
OPERATIONAL IDENTITIES ON GENERALIZED TWO-VARIABLE CHEBYSHEV POLYNOMIALS
}

\author{
C. Cesarano ${ }^{1 \S}$, C. Fornaro ${ }^{2}$ \\ ${ }^{1,2}$ Università Telematica Internazionale Uninettuno \\ Rome, ITALY \\ c.cesarano@uninettunouniversity.net
}

\begin{abstract}
We use the concepts and the formalism of the generalized, $m$-order, two-variable Hermite polynomials of type $H_{n}^{(m)}(x, y)$ in order to derive integral representations of a generalized family of Chebyshev polynomials. Most properties of these polynomials sets can be deduced in a fairly straightforward way from this representation, which actually provides a unifying framework for a large body of polynomials families related to the Gould-Hopper polynomials. It is evident the present generalizations, obtained by using the generalized Hermite polynomials and the integral representation technique, have led to families of Chebyshev polynomials directly related the ordinary case and then we can recognize the generalizations presented in this paper as Chebyshev-like polynomials.
\end{abstract}

AMS Subject Classification: 33C45, 33D45

Key Words: Chebyshev polynomials, Hermite polynomials, Integral representations

\section{Introduction}

There are a number of distinct families of polynomials that go by the name of Chebyshev polynomials $[1,2,3]$. The Chebyshev polynomials par excellence can be defined by:

Received: December 1, 2014

(C) 2015 Academic Publications, Ltd.

$\S$ Correspondence author url: www.acadpubl.eu 
Definition 1. Let $x$ a real variable, we say Chebyshev polynomials of first kind, the polynomials defined by the following relation:

$$
T_{n}(x)=\cos (n \arccos (x))
$$

In the same way we can also introduce the second kind Chebyshev polynomials, by using again the link with the circular functions.

Definition 2. Let $x$ a real variable, we say Chebyshev polynomials of second kind, the polynomials:

$$
U_{n}(x)=\frac{\sin [(n+1) \arccos (x)]}{\sqrt{1-x^{2}}} .
$$

We focus our attention to the Chebyshev polynomials of second kind; we start to observe that by exploiting the relation (2) we can immediately get the follow explicit form:

$$
U_{n}(x)=\sum_{k=0}^{\left[\frac{n}{2}\right]} \frac{(-1)^{k}(n-k) !(2 x)^{n-2 k}}{k !(n-2 k) !}
$$

and the related integral representation has the following form:

$$
U_{n}(x)=\frac{1}{n !} \int_{0}^{+\infty} e^{-t} t^{n} H_{n}\left(2 x,-\frac{1}{t}\right) d t
$$

In some recent papers $[4,5,6]$ dedicated to the Chebyshev polynomials, we have seen interesting integral representations related to ordinary and generalized Chebyshev polynomials itself. The common denominator with respect to which were derived properties are the plethora of identities and operational relations satisfied by the Hermite polynomials in their different forms. In this article, we will investigate families of polynomials that can be traced to the Chebyshev polynomials, always operating with the aid of the Hermite polynomials [7]. By starting from the identities proved for the two-variable Chebyshev polynomials of the form [8]:

$$
U_{n}(x, y)=\sum_{k=0}^{\left[\frac{n}{2}\right]} \frac{(n-k) ! x^{n-2 k} y^{k}}{k !(n-2 k) !}
$$


as for example their integral representation:

$$
U_{n}(x, y)=\frac{1}{n !} \int_{0}^{+\infty} e^{-t} t^{n} H e_{n}\left(x, \frac{y}{t}\right) d t
$$

we will introduce a further family of generalized, two-variable Chebyshev polynomials and we will discuss some interesting relations, with particular regard to their integral representations. The approach is based on generalized, twovariable Hermite polynomials of the form $H_{n}^{(m)}(x, y)$ and related operational relations $[9,10,11]$.

\section{Hermite Polynomials $H_{n}^{(m)}(x, y)$}

It is well known [7] that the two-variable Hermite Polynomials $H_{n}(x, y)$ of Kampé de Fériet form are defined by the following formula:

$$
H_{n}(x, y)=\sum_{r=0}^{\left[\frac{n}{2}\right]} \frac{n !}{r !(n-2 r) !} y^{r} x^{n-2 r} .
$$

In general the above Hermite polynomials can be derived, as a particular case, from a more general class of polynomials recognized as belonging to the Hermite family that we will discuss in this section. To introduce this generalized class of Hermite polynomials we adopt the procedure of the translation operator [12].

Definition 3. We will call Hermite polynomials of the type $H_{n}^{(m)}(x, y)$, the polynomials defined by the formula:

$$
H_{n}^{(m)}(x, y)=\sum_{s=0}^{\left[\frac{n}{m}\right]} \frac{n !}{s !(n-m s) !} y^{s} x^{n-m s} .
$$

It is easy to recognize that the above definition derived from the following relations:

$$
e^{y D^{m}} f(x)=\sum_{n=0}^{+\infty} \frac{y^{n}}{n !} f^{(m n)}(x)
$$

and

$$
D^{m s} x^{n}=n(n-1) \ldots(n-m s+1) x^{n-m s}=\frac{n !}{(n-m s) !} x^{n-m s} .
$$


In fact, for $s=0,1, \ldots\left[\frac{n}{m}\right]$ we obtain:

$$
e^{y D^{m}} x^{n}=\sum_{s=0}^{\left[\frac{n}{m}\right]} \frac{y^{s}}{s !} \frac{n !}{(n-m s) !} x^{n-m s}
$$

that is the explicit form exposed in the Definition 3. It also interesting to note that the Hermite polynomials $H_{n}^{(m)}(x, y)$ can be also introduced by using directly their generating function [13]; in fact by exploiting the exponential funcion $\exp \left(x t+y t^{m}\right)$ we can immediately recognize the identity:

$$
e^{x t+y t^{m}}=\sum_{n=0}^{+\infty} \frac{t^{n}}{n !} H_{n}^{(m)}(x, y)
$$

and, then by setting $m=2$ we obtain the generating function of the Hermite polynomials $H_{n}(x, y)$ (see $\left.(9)\right)$.

Proposition 4. The polynomials $H_{n}^{(m)}(x, y)$ satisfy the following partial differential equation:

$$
\frac{\partial}{\partial y} H_{n}^{(m)}(x, y)=\frac{\partial^{m}}{\partial x^{m}} H_{n}^{(m)}(x, y)
$$

Proof

From (12), by differentiating with respect to $y$, we find:

$$
\sum_{n=0}^{+\infty} \frac{t^{n+m}}{n !} H_{n}^{(m)}(x, y)=\sum_{n=0}^{+\infty} \frac{t^{n}}{n !} \frac{\partial}{\partial y} H_{n}^{(m)}(x, y)
$$

after manipulating the 1.h.s. of the above equation and by equating the like $t-$ powers, we can immediately write:

$$
\frac{n !}{(n-m) !} H_{n-m}^{(m)}(x, y)=\frac{\partial}{\partial y} H_{n}^{(m)}(x, y) .
$$

Otherwise, by deriving $m$-times with respect to $x$ in the (12), we have:

$$
\begin{aligned}
\frac{\partial}{\partial x} H_{n}^{(m)}(x, y) & =n H_{n-1}^{(m)}(x, y) \\
\frac{\partial^{m}}{\partial x^{m}} H_{n}^{(m)}(x, y) & =\frac{n !}{(n-m) !} H_{n-m}^{(m)}(x, y)
\end{aligned}
$$


and then by comparing the second equation of (16) with equation (15), we immediately obtain the partial differential equation (13).

It is worth emphasizing from the previous proof two important recurrence relations related to the polynomials $H_{n}^{(m)}(x, y)$; we have proved, in fact that:

$$
\begin{aligned}
\frac{\partial}{\partial y} H_{n}^{(m)}(x, y) & =\frac{n !}{(n-m) !} H_{n-m}^{(m)}(x, y), \\
\frac{\partial}{\partial x} H_{n}^{(m)}(x, y) & =n H_{n-1}^{(m)}(x, y) .
\end{aligned}
$$

The previous Proposition allows us to derive a similar operational definition for the Hermite polynomials $H_{n}^{(m)}(x, y)$ as in the case of the two-variable Kampé de Fériet polynomials. We note in fact that for $y=0$ in equation (8), we have:

$$
H_{n}^{(m)}(x, 0)=x^{n} .
$$

and, by considering the equation in (13) an ordinary differential equation in the variable $y$, we can immediately conclude that, since it is a linear first order, the solution writes:

$$
H_{n}^{(m)}(x, y)=e^{y \frac{\partial^{m}}{\partial x^{m}}} x^{n}
$$

or, in more explicit terms:

$$
H_{n}^{(m)}(x, y)=\left[\sum_{s=0}^{\left[\frac{n}{m}\right]} \frac{y^{r}}{s !}\left(\frac{\partial}{\partial x}\right)^{m s}\right\rfloor x^{n} .
$$

It is possible to generalize the Burchnall identity $[1,9]$ and the related formulae satisfied by the generalized Hermite polynomials of the type $H_{n}(x, y)$. To obtain the cited results for the generalized Hermite polynomials of order $m$, it is necessary to make some considerations regarding the Weyl identities [14].

Proposition 5. Let $\xi$ be a real parameter, then the following identity holds:

$$
\begin{gathered}
\exp \left(\xi\left(x+\frac{\partial^{n}}{\partial x^{n}}\right)\right)= \\
=\exp \left(x \xi+\frac{\xi^{n+1}}{n+1}\right) \cdot \exp \left(\sum_{r=0}^{(n-1)} \frac{n ! \xi^{r+1}}{(n-r) !(r+1) !}\left(\frac{\partial}{\partial x}\right)^{n-r}\right)
\end{gathered}
$$


Proof

We start to consider this exponential operator:

$$
S(\hat{A}, \hat{B} ; \xi)=e^{\xi\left(\hat{A}+\hat{B}^{n}\right)}
$$

where $\xi$ is a real number and $\hat{A}$ and $\hat{B}$ denotes operators such that:

$$
[\hat{A}, \hat{B}]=\hat{A} \hat{B}-\hat{B} \hat{A}=\hat{k}
$$

with $k$ commuting with both of them. By keeping the derivative of both sides with respect to $\xi$, we get:

$$
\frac{\partial}{\partial \xi} S(\hat{A}, \hat{B} ; \xi)=\left(\hat{A}+\hat{B}^{n}\right) S(\hat{A}, \hat{B} ; \xi)
$$

and, after setting:

$$
S(\hat{A}, \hat{B} ; \xi)=e^{\xi \hat{A}_{\Sigma}}
$$

and by using the relation:

$$
e^{-\xi \hat{A}} \hat{B}^{n} e^{\xi \hat{A}}=(\hat{B}-\xi k)^{n}
$$

we finally find:

$$
\frac{\partial}{\partial \xi} \Sigma=(\hat{B}-\xi k)^{n} \Sigma
$$

which can be easily integrated. Thus getting in conclusion:

$$
S(\hat{A}, \hat{B} ; \xi)=e^{\xi \hat{A}} e^{\sum_{r=0}^{n}\left(\begin{array}{l}
n \\
r
\end{array}\right) \frac{\hat{B}^{n-r} k^{r} \xi^{r+1}}{r+1}(-1)^{r}} .
$$

It is immediately to note that the thesis follows as a particular case with:

$$
\begin{aligned}
& \hat{A}=x \\
& \hat{B}=\frac{\partial}{\partial x}
\end{aligned}
$$

The generalization of the Weyl identity, which we have proved above, allows us to derive the following generalized Burchnall identity:

$$
\left(x+m y \frac{\partial^{m-1}}{\partial x^{m-1}}\right)^{n}=\sum_{r=0}^{n}\left(\begin{array}{l}
n \\
r
\end{array}\right) H_{n-r}^{(m)}(x, y) H_{r}^{(m-1)}(x, y)\left(\{G\}_{s=0}^{m-2}\right)
$$

where we have indicated with $G$ the expression:

$$
G=\frac{m(m-1) ! y}{(m-1-s) !(s+1) !} \frac{\partial^{m-1-s}}{\partial x^{m-1-s}} .
$$


We note that the Burchnall-type identity in (29), for $m=3$, specializes as:

$$
\left(x+3 y \frac{\partial^{2}}{\partial x^{2}}\right)^{2}=\sum_{r=0}^{n}\left(\begin{array}{l}
n \\
r
\end{array}\right) H_{n-r}^{(3)}(x, y) H_{r}\left(3 y \frac{\partial^{2}}{\partial x^{2}}, 3 y \frac{\partial}{\partial x}\right) .
$$

An immediate application of these last identities is the derivation of the following Nielsen formula $[1,15]$ :

$$
H_{2 n}^{(m)}(x, y)=\sum_{r=0}^{n}\left(\begin{array}{l}
n \\
r
\end{array}\right) H_{n-r}^{(m)}(x, y) F_{n, r}^{(m-1)}(x, y)
$$

where

$$
\begin{gathered}
F_{n, r}^{(m-1)}(x, y)= \\
=H_{r}^{(m-1)}\left[\left\{\frac{m(m-1) ! y}{(m-1-r) !(r+1) !} \frac{\partial^{m-1-s}}{\partial x^{m-1-s}}\right\}_{r=0}^{m-2}\right] H_{n}^{(m)}(x, y),
\end{gathered}
$$

and in case $m=3$, we get indeed:

$$
F_{n, r}^{(2)}(x, y)=s ! \sum_{r=0}^{\left[\frac{s}{2}\right]} \frac{(3 y)^{s-r}(2 s-3 r) ! H_{n-(2 s-3 r)}^{(3)}(x, y)}{(s-2 r) ! r ![n-(2 s-3 r)] !} .
$$

A further application of the so far developed method is associated with the derivation of generating functions of the type:

$$
G_{l}^{(m)}(x, y ; t)=\sum_{n=0}^{+\infty} \frac{t^{n}}{n !} H_{n+l}^{(m)}(x, y) .
$$

In fact, by noting that:

$$
e^{\alpha \frac{\partial^{s}}{\partial x_{1}^{s}}} H_{n}^{(m)}\left(x_{1}, \ldots, x_{m}\right)=\left\{\begin{array}{l}
H_{n}^{(m)}\left(x_{1}, \ldots, \alpha+x_{s}, \ldots, x_{m}\right), \\
H_{n}^{(m)}\left(x_{1}, \ldots, x_{m}, \ldots, \alpha\right),
\end{array}\right.
$$

and by using the generalized Burchnall identity (29), we obtain:

$$
G_{l}^{(m)}(x, y ; t)=\sum_{n=0}^{+\infty} \frac{t^{n}}{n !}\left(x+m y \frac{\partial^{m-1}}{\partial x^{m-1}}\right)^{n} H_{l}^{(m)}(x, y)
$$

that is:

$$
G_{l}^{(m)}(x, y ; t)=e^{\left(x+m y \frac{\partial^{m-1}}{\partial x^{m-1}}\right) t} H_{l}^{(m)}(x, y) .
$$

The concepts and the related formalism, presented for the Hermite polynomials of type $H_{n}^{(m)}(x, y)$, will be useful to derive many important relations regarding the generalized family of two-variable Chebyshev polynomials that we are preparing to discuss. 


\section{Generalized Two-Variable Chebyshev Polynomials}

As we have mentioned in the introduction, in a previous article [8], we have discussed the generalized two-variable Chebyshev polynomials of the type $U_{n}(x, y)$, and we have derived many relevat relations. Now, we want introduce an extension of this class of generalized, two-variable Chebyshev polynomials by using the structure and the related properties of the $m$-order Hermite polynomials presented in the previous section. By expanding what we have seen previously, relevant to the Chebyshev polynomials $U_{n}(x, y)$, we will discuss the Chebyshev polynomials in several indices and several variables. It could be interesting to note that we have already presented, in the paper [6], a different generalization of the Chebyshev polynomials in the multi-index way, by using the tool of the Hermite polynomials of the form $H_{n}^{(m)}(x, y)$. We remind, in fact that the polynomials of type:

$$
{ }_{m} U_{n}(x, y ; \alpha)=\frac{1}{n !} \int_{0}^{+\infty} e^{-\alpha t} t^{n} H_{n}^{(m)}\left(m x,-\frac{y}{t^{m-1}}\right) d t,
$$

are called generalized, $m$-order, two-variable, second kind Chebyshev polynomials, and their generating function is given by:

$$
\sum_{n=0}^{+\infty} \xi^{n}\left[{ }_{m} U_{n}(x, y ; \alpha)\right]=\frac{1}{\alpha-m x \xi+y \xi^{m}}
$$

where $\xi \in \mathbb{R},|\xi|<1$.

We can directly define the further family of multi-index, generalized Chebyshev polynomials, by acting on the structure of the Chebyshev polynomials of type $U_{n}(x, y)$ and subsequently, we estabilish the relation with the Hermite polynomials of type $H_{n}^{(m)}(x, y)$.

Definition 6. We will call generalized two-variable $m$-order Chebyshev polynomials, the polynomials defined by the formula:

$$
U_{n}^{(m)}(x, y)=\sum_{k=0}^{\left[\frac{n}{m}\right]} \frac{(n-k) ! x^{n-m k} y^{k}}{k !(n-m k) !}
$$

where $x, y \in \mathbb{R}$ and $n, m \in \mathbb{N}$.

By using the Hermite polynomials $H_{n}^{(m)}(x, y)$ we can immediately derive the integral representation for the Chebyshev polynomials of the type $U_{n}^{(m)}(x, y)$. 
Proposition 7. The generalized Chebyshev polynomials $U_{n}^{(m)}(x, y)$ satisfy the following integral representation:

$$
U_{n}^{(m)}(x, y)=\frac{1}{n !} \int_{0}^{+\infty} e^{-t} t^{n} H_{n}^{(m)}\left(x, \frac{y}{t}\right) d t .
$$

Proof

Since:

$$
(n-k) !=\int_{0}^{+\infty} e^{-t} t^{n-k} d t
$$

we can write, from the identity (41):

$$
U_{n}^{(m)}(x, y)=\sum_{k=0}^{\left[\frac{n}{m}\right]} \int_{0}^{+\infty} e^{-t} t^{n-k} \frac{x^{n-m k} y^{k}}{k !(n-m k) !} d t
$$

and, by manipulating the r.h.s. of the above relation, we find:

$$
U_{n}^{(m)}(x, y)=\int_{0}^{+\infty} e^{-t} t^{n} \sum_{k=0}^{\left[\frac{n}{m}\right]} \frac{x^{n-m k}}{k !(n-m k) !}\left(\frac{y}{t}\right)^{k} d t
$$

Finally, from the explicit form of the polynomials $H_{n}^{(m)}(x, y)$, (see eq. (8)), immediately follows the thesis.

We will use the structure and the properties of the Hermite polynomials of the type $H_{n}^{(m)}(x, y)$, to explore the nature and the characteristics of the present Chebyshev polynomials $U_{n}^{(m)}(x, y)$.

Proposition 8. Let $\xi$ a real number such that $|\xi|<1$; the generalized Chebyshev polynomials $U_{n}^{(m)}(x, y)$ admit the follow generating function:

$$
\sum_{n=0}^{+\infty} \xi^{n} U_{n}^{(m)}(x, y)=\int_{0}^{+\infty} e^{-t(1-x \xi)} e^{y \xi^{m} t^{m-1}} d t .
$$

Proof

In the eq. (12) we heve stated the expression of the generating function of the polynomials $H_{n}^{(m)}(x, y)$ :

$$
e^{x t+y t^{m}}=\sum_{n=0}^{+\infty} \frac{t}{n !} H_{n}^{(m)}(x, y)
$$


From the integral representation of the Chebyshev polynomials $U_{n}^{(m)}(x, y)$, (see eq.(42)), after multiplying by $\xi^{n}$, with $|\xi|<1$ and summing up over $n$, we obtain:

$$
\sum_{n=0}^{+\infty} \xi^{n} U_{n}^{(m)}(x, y)=\sum_{n=0}^{+\infty} \frac{\xi^{n}}{n !} \int_{0}^{+\infty} e^{-t} t^{n} H_{n}^{(m)}\left(x, \frac{y}{t}\right) d t
$$

The above relation can be rearranged in the form:

$$
\sum_{n=0}^{+\infty} \xi^{n} U_{n}^{(m)}(x, y)=\int_{0}^{+\infty} e^{-t} \sum_{n=0}^{+\infty} \frac{(\xi t)^{n}}{n !} H_{n}^{(m)}\left(x, \frac{y}{t}\right) d t
$$

and then from (42), we have:

$$
\sum_{n=0}^{+\infty} \xi^{n} U_{n}^{(m)}(x, y)=\int_{0}^{+\infty} e^{-t} e^{(x t) \xi+\left(y t^{m-1}\right) \xi^{m}} d t
$$

The above identity is formally the statement (44).

It is important to note that the integral:

$$
\int_{0}^{+\infty} e^{-t(1-x \xi)} e^{y \xi^{m} t^{m-1}} d t
$$

diverges when $y>0$. Otherwise for the values of $m$ greather than 2 and $y<0$, the integal representation of the polynomials $U_{n}^{(m)}(x, y)$ can be written in the form:

$$
\sum_{n=0}^{+\infty} \xi^{n} U_{n}^{(m)}(x, y)=\frac{1}{1+x \xi+y \xi^{m}}
$$

In the previous section, we have stated the important recurrence relations for the Hermite polynomials $H_{n}^{(m)}(x, y)$, (see eqs. (16)), which can be customed in the form:

$$
\begin{gathered}
\frac{\partial}{\partial x} H_{n}^{(m)}\left(x, \frac{y}{t}\right)=n H_{n-1}^{(m)}\left(x, \frac{y}{t}\right) \\
\left(x+m \frac{y}{t} \frac{\partial^{m-1}}{\partial x^{m-1}}\right) H_{n}^{(m)}\left(x, \frac{y}{t}\right)=H_{n+1}^{(m)}\left(x, \frac{y}{t}\right) .
\end{gathered}
$$

These relations can be used to state the recurrence relations involving the generalized Chebyshev polynomials $U_{n}^{(m)}(x, y)$. 
Proposition 9. The generalized Chebyshev polynomials of the type $U_{n}^{(m)}(x, y)$ satisfy the following identities:

$$
\begin{gathered}
m y \frac{\partial^{m-1}}{\partial x^{m-1}} U_{n-1}^{(m)}(x, y)=\left(n-x \frac{\partial}{\partial x}\right) U_{n}^{(m)}(x, y), \\
U_{n+1}^{(m)}(x, y)=x U_{n}^{(m)}(x, y)+(m-1) y \frac{\partial^{m-2}}{\partial x^{m-2}} U_{n-1}^{(m)}(x, y) .
\end{gathered}
$$

Proof

From the integral representation of the Chebyshev polynomials $U_{n}^{(m)}(x, y)$, stated in the (42), by substituting the multiplicative recurrence relation related to the Hermite polynomials $H_{n}^{(m)}\left(x, \frac{y}{t}\right)$, (eq. (48)), we have:

$$
U_{n}^{(m)}(x, y)=\frac{1}{n !} \int_{0}^{+\infty} e^{-t} t^{n}\left(x+m \frac{y}{t} \frac{\partial^{m-1}}{\partial x^{m-1}}\right) H_{n-1}^{(m)}\left(x, \frac{y}{t}\right) d t .
$$

The r.h.s. of the above identy can be exploited to give:

$$
\begin{aligned}
U_{n}^{(m)}(x, y)= & \frac{x}{n !} \int_{0}^{+\infty} e^{-t} t^{n} H_{n-1}^{(m)}\left(x, \frac{y}{t}\right) d t+ \\
& +\frac{m y}{n !} \int_{0}^{+\infty} e^{-t} t^{n-1} \frac{\partial^{m-1}}{\partial x^{m-1}} H_{n-1}^{(m)}\left(x, \frac{y}{t}\right) d t
\end{aligned}
$$

and then:

$$
\begin{aligned}
& U_{n}^{(m)}(x, y)-\frac{x}{n(n !)} \int_{0}^{+\infty} e^{-t} t^{n} \frac{\partial}{\partial x} H_{n}^{(m)}\left(x, \frac{y}{t}\right) d t= \\
& =\frac{m y}{n(n-1) !} \int_{0}^{+\infty} e^{-t} t^{n-1} \frac{\partial^{m-1}}{\partial x^{m-1}} H_{n-1}^{(m)}\left(x, \frac{y}{t}\right) d t .
\end{aligned}
$$

It is to recognize in the above relation the first of the identities in the statement.

We have stated, for the two-variable Chebyshev polynomials of type $U_{n}(x, y)$, see [8], the following relation:

$$
U_{n+1}(x, y)=x U_{n}(x, y)+y U_{n-1}(x, y) .
$$

Then, to prove the second identity of the present proposition, we can use the induction over $m$, by noting that it is a formally extension of the above mentioned recurrence relation related to the polynomials $U_{n}(x, y)$. 
The relations obtained in the above result can be used to better clarify the recurrence of the polynomials $U_{n}^{(m)}(x, y)$. We note in fact that the second of (49) can be written as:

$$
U_{n+1}^{(m)}(x, y)=x U_{n}^{(m)}(x, y)+(m-1) y \widehat{D}_{x}^{-1} \frac{\partial^{m-1}}{\partial x^{m-1}} U_{n-1}^{(m)}(x, y)
$$

and by using the first of (49), we get:

$$
U_{n+1}^{(m)}(x, y)=x U_{n}^{(m)}(x, y)+\frac{m-1}{m} \widehat{D}_{x}^{-1}\left(n-x \frac{\partial}{\partial x}\right) U_{n}^{(m)}(x, y) .
$$

The above identity and the first of the (49), allow us to define the rising and lowering operators related to the generalized Chebyshev polynomials $U_{n}^{(m)}(x, y)$, by setting:

$$
\begin{gathered}
\widehat{E}_{+}=x+\frac{m-1}{m} \widehat{D}_{x}^{-1}\left(n-x \frac{\partial}{\partial x}\right) \\
\widehat{E}_{-}=\frac{1}{m y} \widehat{D}_{x}^{-(m-1)}\left(n-x \frac{\partial}{\partial x}\right)
\end{gathered}
$$

which, since we have noted before, act on the polynomials $U_{n}^{(m)}(x, y)$ as follows:

$$
\begin{aligned}
& \widehat{E}_{+} U_{n}^{(m)}(x, y)=U_{n+1}^{(m)}(x, y) \\
& \widehat{E} \__{-} U_{n}^{(m)}(x, y)=U_{n-1}^{(m)}(x, y) .
\end{aligned}
$$

We can use these relations to derive the differential equation satisfied by the generalized two-variable Chebyshev polynomials of type $U_{n}^{(m)}(x, y)$.

Theorem 10. The polynomials $U_{n}^{(m)}(x, y)$ satisfy the following partial differential equation:

$$
\begin{gathered}
{\left[m y \frac{\partial^{m}}{\partial x^{m}}+\frac{x^{2}}{m} \frac{\partial^{2}}{\partial x^{2}}+\left(1+n-\frac{2 n-1}{m}\right) x \frac{\partial}{\partial x}-n\left(1+\frac{n(m-1)}{m}\right)\right] .} \\
\cdot U_{n}^{(m)}(x, y)=0
\end{gathered}
$$

Proof

By using the structure of the rising and lowering operators defined above, we can immediately write the relation:

$$
\widehat{E}_{-} \widehat{E}_{+} U_{n}^{(m)}(x, y)=U_{n}^{(m)}(x, y)
$$


which in an explicit form, reads:

$$
\begin{gathered}
{\left[\frac{1}{m y} \widehat{D}_{x}^{-(m-1)}\left((n+1)-x \frac{\partial}{\partial x}\right)\right]\left[x+\frac{m-1}{m} \widehat{D}_{x}^{-1}\left(n-x \frac{\partial}{\partial x}\right)\right] .} \\
\cdot U_{n}^{(m)}(x, y)=U_{n}^{(m)}(x, y)
\end{gathered}
$$

and then:

$$
\begin{gathered}
\widehat{D}_{x}^{-(m-1)}\left((n+1)-x \frac{\partial}{\partial x}\right)\left[x+\frac{m-1}{m} \widehat{D}_{x}^{-1}\left(n-x \frac{\partial}{\partial x}\right)\right] U_{n}^{(m)}(x, y)= \\
=m y U_{n}^{(m)}(x, y) .
\end{gathered}
$$

It is easy note that the follow operational identities hold:

$$
\begin{aligned}
& \frac{\partial^{m-1}}{\partial x^{m-1}} \widehat{D}_{x}^{-(m-1)}=\widehat{1} \\
& \widehat{D}_{x}^{-(m-1)} \frac{\partial^{m-1}}{\partial x^{m-1}}=\widehat{1}
\end{aligned}
$$

and then, by deriving $m$-times with respect to $x$ in the equation (56), we obtain:

$$
\begin{gathered}
\left((n+1)-x \frac{\partial}{\partial x}\right)\left[x+\frac{m-1}{m} \widehat{D}_{x}^{-1}\left(n-x \frac{\partial}{\partial x}\right)\right] U_{n}^{(m)}(x, y)= \\
=m y \frac{\partial^{m-1}}{\partial x^{m-1}} U_{n}^{(m)}(x, y) .
\end{gathered}
$$
write:

$$
\begin{aligned}
m y \frac{\partial^{m-1}}{\partial x^{m-1}}= & \left((n+1)-x \frac{\partial}{\partial x}\right)\left[x+\frac{m-1}{m} \widehat{D}_{x}^{-1}\left(n-x \frac{\partial}{\partial x}\right)\right] \\
= & (n+1) x+(n+1) \frac{m-1}{m} \widehat{D}_{x}^{-1}\left(n-x \frac{\partial}{\partial x}\right)-x \frac{\partial}{\partial x} x+ \\
& -x \frac{m-1}{m}\left(n-x \frac{\partial}{\partial x}\right)
\end{aligned}
$$

and by deriving again with respect to $x$, we get:

$$
m y \frac{\partial^{m}}{\partial x^{m}}=(n+1) \frac{\partial}{\partial x} x+(n+1) \frac{m-1}{m}\left(n-x \frac{\partial}{\partial x}\right)-\frac{\partial}{\partial x} x \frac{\partial}{\partial x} x+
$$




$$
-\frac{\partial}{\partial x} x \frac{m-1}{m}\left(n-x \frac{\partial}{\partial x}\right) .
$$

By noting that the follow identities hold:

$$
\begin{aligned}
\frac{\partial}{\partial x} x & =1+x \frac{\partial}{\partial x} \\
\frac{\partial}{\partial x} x^{2} \frac{\partial}{\partial x} & =2 x \frac{\partial}{\partial x}+x^{2} \frac{\partial^{2}}{\partial x^{2}}
\end{aligned}
$$

we can rearranged the identity (58) in the form:

$$
\begin{gathered}
m y \frac{\partial^{m}}{\partial x^{m}}=(n+1)\left(1+x \frac{\partial}{\partial x}\right)+\frac{(n+1)(m-1)}{m}\left(n-x \frac{\partial}{\partial x}\right)+ \\
-\frac{\partial}{\partial x} x\left(1+x \frac{\partial}{\partial x}\right)+\frac{n(m-1)}{m} \frac{\partial}{\partial x} x+\frac{m-1}{m} \frac{\partial}{\partial x} x^{2} \frac{\partial}{\partial x}
\end{gathered}
$$

then:

$$
\begin{gathered}
m y \frac{\partial^{m}}{\partial x^{m}}=(n+1)+(n+1) x \frac{\partial}{\partial x}+\frac{n(n+1)(m-1)}{m}-\frac{(n+1)(m-1)}{m} x \frac{\partial}{\partial x}+ \\
-\frac{\partial}{\partial x} x-\frac{\partial}{\partial x} x^{2} \frac{\partial}{\partial x}-\frac{n(m-1)}{m}-\frac{n(m-1)}{m} x \frac{\partial}{\partial x}+\frac{m-1}{m} \frac{\partial}{\partial x} x^{2} \frac{\partial}{\partial x}
\end{gathered}
$$

and finally:

$$
m y \frac{\partial^{m}}{\partial x^{m}}=-\frac{x^{2}}{m} \frac{\partial^{2}}{\partial x^{2}}+\left(-n-1+\frac{2 n-1}{m}\right) x \frac{\partial}{\partial x}+n\left(1+\frac{n(m-1)}{m}\right) .
$$

By substituting the above relation in the (57) we immediately obtain the thesis.

In this paper, we have presented the generalized, two-variable Chebyshev polynomials of second kind of type $U_{n}^{(m)}(x, y)$ and we have discussed, in particular, some interested integral representations. We have deduced some relevant properties by using the structure and the operational relations satisfied by the Hermite polynomials of the form $H_{n}^{(m)}(x, y)$, introduced in the second section.

It has been outlined in the abstract that these families of polynomials could be indicated as Chebyshev-like polynomials, since they present a substantial generalization of the ordinary Chebyshev polynomials; a fortiori, the polynomials defined through the relation:

$$
U_{n}^{(m)}(x, y)=\sum_{k=0}^{\left[\frac{n}{m}\right]} \frac{(n-k) ! x^{n-m k} y^{k}}{k !(n-m k) !}
$$


and the related properties shown, further emphasize the different nature than the ordinary Chebyshev polynomials, while on the contrary prove their connection with generalized Hermite polynomials $H_{n}^{(m)}(x, y)$ used to derive their properties. In a forthcoming paper, we will show further integral representations for the Chebyshev-like polynomials by using, again, the properties of some special Hermite polynomials, but also with the help of a generalized class of exponential truncated polynomials [16] and some particular generalizations of Hermite and Humbert polynomials [17, 18]

\section{References}

[1] G.E. Andrews, R. Askey and R. Roy, Special Functions, Encyclopedia of Mathematics and its Applications 71, Cambridge University Press, United Kingdom (1999).

[2] G. Dattoli, D. Sacchetti and C. Cesarano, A note on Chebyshev polynomials, Annali dell'Università di Ferrara. Sezione 7: Scienze matematiche, XLVII (2001), 107-115.

[3] G. Dattoli, C. Cesarano and S. Lorenzutta, From Hermite to Humbert Polynomials, Rendiconti dell'Istituto di matematica dell'Università di Trieste, XXXV (2003), 37-48.

[4] C. Cesarano, Identities and generating functions on Chebyshev polynomials, Georgian Mathematical Journal, 19 (2012), 427-440.

[5] C. Cesarano, Generalized Chebyshev polynomials, HACETTEPE Journal of Mathematics and Statistics, 43(5) (2014), 731-740.

[6] C. Cesarano, Generalization of two-variable Chebyshev and Gegenbauer polynomials, International Journal of Applied Mathematics and Statistics, 53(1) (2015), 1-7.

[7] P. Appell and J. Kampé de Fériet, Fonctions hypergéométriques et hypersphériques. Polynômes d'Hermite, Gauthier-Villars, Paris, France (1926).

[8] C. Cesarano and C. Fornaro, A note on two-variable Chebyshev polynomials, Georgian Mathematical Journal, accepted for publication (2015).

[9] H.W. Gould, A.T. Hopper, Operational formulas connected with two generalizations of Hermite Polynomials, Duke Mathematics Journal, 29 (1962), 51-62. 
[10] G. Dattoli and C. Cesarano, On a new family of Hermite polynomials associated to parabolic cylinder functions, Applied Mathematics and Computation, 141 (2003), 143-149.

[11] G. Dattoli P.E. Ricci and C. Cesarano, A Note on Multi-index Polynomials of Dickson Type and their Applications in Quantum Optics, Journal of Computational and Applied Mathematics, 145 (2002), 417-424.

[12] G. Dattoli, S. Lorenzutta and C. Cesarano, Generalized Polynomials and New Families of Generating Functions, Annali dell'Università di Ferrara. Sezione \%: Scienze matematiche, XLVII (2001), 57-61.

[13] H.M. Srivastava, H.L. Manocha, A treatise on generating functions, Wiley, New York, (1984).

[14] J.F. Adams, Lectures on Lie Groups, Chicago Lectures in Mathematics, University of Chicago Press (1969).

[15] L.C. Andrews, Special Functions for Applied Matematicians and Engineers, Mc Millan, New York (1985).

[16] G. Dattoli, C. Cesarano and D. Sacchetti, A Note on Truncated Polynomials, Applied Mathematics and Computation, 134 (2003), 595-605.

[17] C. Cesarano, G.M. Cennamo and L. Placidi, Humbert Polynomials and Functions in Terms of Hermite Polynomials Towards Applications to Wave Propagation, WSEAS Transactions on Mathematics, 13 (2014), 595-602.

[18] G. Dattoli, S. Lorenzutta and C. Cesarano, Bernestein polynomials and operational methods, Journal of Computational Analysis and Applications, 8 (2006), 369-377. 